\title{
La fréquentation des dispositifs d'aide aux élèves en difficulté au cours préparatoire : un révélateur de l'organisation scolaire contemporaine
}

Intervention programs for first-grade struggling readers: an illustration of contemporary school organisation

\section{Céline Piquée et Caroline Viriot-Goeldel}

\section{OpenEdition Journals}

Édition électronique

URL : http://journals.openedition.org/rfp/4975

DOI : $10.4000 /$ rfp.4975

ISSN : 2105-2913

Éditeur

ENS Éditions

Édition imprimée

Date de publication : 31 mars 2016

Pagination : 47-70

ISSN : 0556-7807

Référence électronique

Céline Piquée et Caroline Viriot-Goeldel, «La fréquentation des dispositifs d'aide aux élèves en difficulté au cours préparatoire : un révélateur de l'organisation scolaire contemporaine », Revue française de pédagogie [En ligne], 194 | 2016, mis en ligne le 01 juin 2020, consulté le 04 janvier 2021. URL : http://journals.openedition.org/rfp/4975 ; DOI : https://doi.org/10.4000/rfp.4975 


\title{
La fréquentation des dispositifs d'aide aux élèves en difficulté au cours préparatoire : un révélateur de l'organisation scolaire contemporaine
}

\author{
Céline Piquée \\ Caroline Viriot-Goeldel
}

\begin{abstract}
La présente recherche décrit l'admission des élèves en difficulté en lecture/écriture au sein de différents dispositifs d'aide au cours préparatoire. À partir d'un échantillon de 2507 élèves scolarisés dans 131 classes, il apparaît qu'un nombre important d'élèves sont aidés par l'institution scolaire (un tiers, tous dispositifs confondus), mais aussi qu'ils bénéficient d'un faible volume horaire d'aide en général. La recherche souligne également le recours aux orthophonistes pour $12 \%$ d'élèves. Une analyse plus approfondie permet de caractériser les publics aidés, révélant ainsi une relative inadéquation au niveau des élèves. L'ensemble des résultats rend compte de la reconfiguration contemporaine de l'organisation scolaire, dont les mots d'ordre sont individualisation, externalisation et médicalisation.
\end{abstract}

Mots-clés (TESE) : enseignement primaire, difficulté de lecture, soutien pédagogique, sociologie de l'éducation.

En France, plusieurs recherches nationales et internationales estiment à près de $15 \%$ le nombre d'élèves éprouvant des difficultés de lecture importantes à l'issue de l'école primaire (International Association for the Evaluation of Educational Achievement [IEA], 2012) : ils sont capables de prélever des informations dans un texte court si elles sont explicites, mais ils sont en difficulté face à des tâches plus complexes (Gibert, Levasseur \& Pastor, 2004; Colmant, Daussin \& Bessonneau, 2011), notamment parce que leur procédure de reconnaissance des mots est lente et leur vocabulaire peu étendu (Bonjour \& Gombert, 2004). Le constat est similaire au niveau du secondaire (OCDE, 2014). Ces recherches attestent également un fort déterminisme social précoce de la réussite scolaire et une dégradation globale régulière des performances en lecture et des acquis des élèves les plus fragiles au cours de ces quinze à vingt dernières années (Rocher, 2008; Rocher \& Le Donné, 2012; OCDE, 2014).

On comprend alors que «la prévention de l'illettrisme figure au cœur des missions de l'éducation et a lieu d'abord dans le cadre de la classe, dans les écoles» 
(Ministère de l'Éducation nationale et de I'Enseignement supérieur et de la Recherche [MENESR], 2013a), et la succession des plans de prévention de l'illettrisme témoigne de l'importance de cette question aux yeux de l'institution elle-même (Ferry, Darcos \& Haigneré, 2003 ; Chatel, 2010; MENESR, 2013a). Ces plans visent à «mener une action volontariste au cœur de l'École» afin de prévenir les difficultés de lecture et de permettre à l'école de la République «d'apprendre à lire, à écrire et à compter à tous les enfants de France» (Chatel, 2010). Plusieurs de leurs mesures concernent le cours préparatoire, niveau auquel débute en France l'enseignement formel de la lecture. En effet, ce niveau joue un rôle décisif dans l'acquisition des compétences de lecture, et l'institution elle-même déclare qu'«en ce qui concerne la lecture, il est absolument essentiel que les élèves en aient une maîtrise suffisante dès la fin du $\mathrm{CP}$, le retard pris à ce moment-là étant quasiment prédictif de l'échec scolaire» (Haut Conseil de l'Éducation [HCE], 2012, p. 16).

Un enseignement de la lecture efficace dès le cours préparatoire constitue sans doute la meilleure prévention des difficultés de lecture (Snow, Burns \& Griffin, 1998). C'est ainsi que diverses modélisations des parcours d'aide l'instituent comme le premier niveau, la «pierre angulaire» des apprentissages, par exemple la modélisation américaine en trois tiers (Fuchs \& Fuchs, 2006), mise en œuvre également dans plusieurs provinces canadiennes (Ministère de l'Éducation, du Loisir et du Sport [MELS], 2011). Une évaluation régulière des progrès des élèves à l'aide d'outils standardisés comportant des seuils de performances en deçà desquels les progrès sont jugés insuffisants permet de proposer aux élèves une aide d'intensité croissante au sein de trois niveaux. Le premier niveau renvoie à l'enseignement en classe, y compris l'aide apportée par le maître aux élèves en difficulté au sein de la classe. Le deuxième niveau s'adresse aux élèves qui ne progressent pas suffisamment dans ce cadre et concerne donc toute aide supplémentaire apportée soit par le maître hors temps scolaire, soit par des dispositifs dits de «niveau 2 » (aide en petit groupe dispensée par un enseignant spécialisé). Enfin, dans un dernier temps interviennent des aides dites de «niveau 3 » (aide intensifiée selon diverses modalités : individualisation, augmentation de la fréquence et de la durée de l'aide, recours à d'autres types de spécialistes, etc.; Haager, Klinger \& Vaughn, 2007).

Un des principes à la base de ces dispositifs d'aide mentation du temps d'engagement de l'élève dans les activités. Le temps d'engagement est défini par la quantité de temps durant laquelle l'élève s'engage activement dans l'apprentissage (Caroll, 1963, cité par Chopin, 2010). Il diffère du temps passé sur un contenu d'enseignement en retirant à ce dernier à la fois les interruptions diverses liées le plus souvent à la gestion de la classe mais également les temps d'inattention de l'élève'. D'une manière générale, de nombreuses recherches établissent que le temps d'enseignement est relié positivement à la réussite scolaire des élèves (Suchaut, 1996; Crahay, 2012). Le constat selon lequel les élèves apprennent d'autant mieux que le temps consacré à l'apprentissage est élevé, mais surtout que le temps d'engagement des élèves dans les apprentissages est élevé, vaut également pour la lecture. Suchaut, Bougnères et Bouguen (2014) estiment ainsi à 35 heures le temps d'engagement effectif de l'élève sur les tâches d'apprentissage de la lecture (ALT, pour Academic Learning Time) nécessaire pour atteindre les compétences attendues en lecture, alors que les élèves de leur échantillon ne bénéficient que de 20 heures en moyenne. II manque donc $15 \mathrm{~h}$ d'ALT pour permettre aux élèves les plus faibles d'atteindre un niveau satisfaisant en lecture à la fin du CP. Dans ce contexte, les stratégies visant à optimiser le temps scolaire nécessaire aux besoins des élèves consistent à augmenter le temps d'engagement des élèves à travers une aide individualisée (travail en petit groupe, face-à-face pédagogique...). C'est ce que proposent les dispositifs dont il sera question ici.

En France, au-delà des aides que les enseignants apportent à leurs élèves dans le cadre de la classe, l'aide aux élèves en difficulté est d'abord dispensée dans le cadre des Activités pédagogiques complémentaires (APC). Ces activités ont été mises en place à la rentrée 2013 à raison d'un volume horaire total de 36 heures annuelles par classe, "soit pour aider les élèves lorsqu'ils rencontrent des difficultés dans leurs apprentissages, soit pour les accompagner dans leur travail personnel ou leur proposer toute autre activité prévue par le projet d'école, le cas échéant en lien avec le projet éducatif territorial » (MENESR, 2013b). Les APC offrent ainsi aux élèves qui en bénéficient un temps

1 La mesure du temps d'engagement nécessite des dispositifs empiriques assez lourds basés sur des observations de classes. Elle n'est cependant jamais tout à fait parfaite puisqu'un élève peut présenter des signes extérieurs d'implication dans la tâche sans pour autant être vraiment engagé cognitivement. Pour des développements sur les usages du temps dans les recherches sur l'enseignement, voir Chopin, 2010. 
d'enseignement supplémentaire, temps individuel ou en petit groupe.

Dans la mesure où «l'aide apportée par l'enseignant [...] peut ne pas suffire pour certains élèves» (MENESR, 2014a), des aides spécialisées peuvent également être apportées par les Réseaux d'aides spécialisées aux élèves en difficulté (RASED), selon un principe de subsidiarité semblable à celui du modèle en trois niveaux. Au sein des RASED, les maîtres E ont pour mission d'apporter «une aide aux élèves qui ont des difficultés avérées à comprendre et à apprendre dans le cadre des activités scolaires. Il s'agit, pour l'enseignant spécialisé, de prévenir et de repérer, grâce à une analyse partagée avec l'enseignant de la classe ou l'équipe pédagogique du cycle, les difficultés d'apprentissage de ces élèves et d'apporter une remédiation pédagogique dans le cadre d'un projet d'aide spécialisée » (MENESR, 2014a). Les maîtres G sont quant à eux chargés de l'aide spécialisée à dominante rééducative qui « apporte une aide aux élèves dont l'analyse de la situation montre qu'il faut faire évoluer leurs rapports aux exigences de l'école, instaurer ou restaurer I'investissement dans les activités scolaires. Il s'agit, pour l'enseignant spécialisé, de prévenir et repérer, grâce à une analyse partagée avec l'enseignant de la classe ou l'équipe pédagogique du cycle, les difficultés de comportement de ces élèves et de mettre en œuvre des actions, dans le cadre d'un projet d'aide spécialisée, pour faire évoluer les situations »(MENESR, 2014a). Contrairement aux APC, I'intervention du RASED prend place dans le temps scolaire; elle est alors susceptible de concurrencer l'enseignement du maître dans sa classe. Son organisation individuelle ou en petit groupe doit cependant permettre d'optimiser l'engagement des élèves pendant des activités spécifiques.

Enfin, en France, plusieurs travaux révèlent qu'une partie du soutien en lecture est apportée hors de l'école par les orthophonistes, spécialistes des troubles du langage oral ou écrit (Garcia, 2013; Morel, 2014; Viriot-Goeldel, 2013). Le recours à des séances d'orthophonie semblerait donc être devenu un moyen ordinaire de remédiation aux difficultés d'apprentissage de la lecture, bien que nous ne disposions pas de chiffres qui permettraient d'appréhender l'ampleur du phénomène. La formation de ces professionnels du secteur paramédical dépend des facultés de médecine, le bilan orthophonique se fait sur prescription médicale et les séances sont prises en charge par la sécurité sociale. Les parents consultent les orthophonistes en libéral ou dans des centres médico-psychopédagogiques (CMPP).
D'une manière générale, on sait peu de chose sur l'ensemble de ces dispositifs d'aide. On ignore combien d'élèves en bénéficient et à hauteur de combien de temps (Cour des comptes, 2015). En l'absence de protocole précis pour désigner les bénéficiaires de ces aides, on ne sait pas non plus quelles sont les caractéristiques sociodémographiques et scolaires des élèves aidés. Il est alors malaisé d'apprécier dans quelle mesure ces dispositifs s'adressent effectivement aux élèves les plus faibles et offrent un temps d'apprentissage supplémentaire comparable à ceux dont la recherche a montré qu'ils influençaient positivement les progrès des élèves. C'est pourquoi cette étude vise à examiner empiriquement la manière dont ces aides sont distribuées aux élèves de cours préparatoire. Au-delà d'une description globale de la quantité d'élèves aidés et de l'intensité de l'aide dispensée, elle interroge donc l'adéquation entre le public visé par les dispositifs et le public qui y est effectivement accueilli.

Après avoir présenté la méthodologie de l'enquête et les données recueillies, deux grandes questions guideront notre analyse : quelles sont l'ampleur et l'intensité du recours aux dispositifs d'aide aux élèves en difficulté au cours préparatoire? Quelles sont les caractéristiques sociodémographiques et scolaires des élèves qui y sont accueillis, comparativement notamment à ceux qui n'y sont pas accueillis? Dans une dernière partie, l'ensemble des résultats obtenus sera discuté au regard de ce qu'ils révèlent du fonctionnement des dispositifs d'aide aux élèves en difficulté : capacité à offrir un temps d'apprentissage supplémentaire aux élèves les plus en difficulté et respect du principe de subsidiarité. Plus largement, les résultats seront aussi discutés au regard de ce qu'ils révèlent des transformations de l'école : individualisation, externalisation et médicalisation ${ }^{2}$ sont les mots d'ordre de la lutte contre l'échec scolaire au sein d'une redéfinition de l'organisation scolaire traditionnelle.

\section{L'enquête}

Les données analysées ici sont issues de la recherche "Lire-Écrire à l'école primaire ${ }^{3}$. Cette recherche

2 Avec Morel, nous définissons la médicalisation comme un "processus qui conduit un élève en difficulté dans le cadre scolaire à être appréhendé en tant qu'individu nécessitant des "soins" et à consulter [...] des "professionnels du soin" qui appréhendent l'élève en difficulté comme un "patient"» (2014, p. 19).

3 Cette recherche, coordonnée par I'Institut français de l'Éducation [IFE], est financée notamment par la DGESCO et l'IFE. 
(Goigoux, 2016) étudie l'influence des pratiques d'enseignement de la lecture et de l'écriture sur la qualité des premiers apprentissages. S'agissant d'identifier les caractéristiques didactiques et pédagogiques les plus efficaces et les plus équitables, elle interroge, entre autres, les pratiques d'aide aux élèves faibles dans la classe et hors de cette dernière. Nous ne présentons ici que les éléments de méthodologie en lien avec la question de l'aide apportée aux élèves en difficulté dans l'apprentissage de la lecture.

\section{Participants}

Durant l'année 2013-2014, 131 classes accueillant 2507 élèves de cours préparatoire, réparties sur l'ensemble du territoire français, ont participé à l'enquête. Les enseignants ont été choisis pour rendre compte d'une diversité de contextes sociaux et géographiques ainsi que d'une variété de styles didactiques et pédagogiques. Dans la mesure où nous voulions évaluer l'impact de leurs techniques didactiques, nous avons choisi des maîtres expérimentés : ils ont tous au moins trois ans d'expérience au cours préparatoire. Le protocole de recueil de données nécessitant la capture vidéo de séances de classe observées, les enseignants n'ont pas été choisis aléatoirement mais sur la base du volontariat. Notre échantillon n'affiche cependant pas de grands écarts par rapport à la population des écoles françaises. Les classes comptent 22,2 élèves en moyenne (écart-type $=3,6$ ), contre 22,9 pour la moyenne nationale (MENESR, 2014b). L'ancienneté moyenne des enseignants y est de 16,4 ans (écart-type $=7,4)$, alors qu'elle est estimée à 15,8 ans pour la moyenne nationale (Gambert \& Bonneau, 2005), et de 8,4 ans au cours préparatoire (écart-type $=5,3$ ). On note toutefois une surreprésentation de l'éducation prioritaire (environ $30 \%$ dans l'échantillon contre $20 \%$ au niveau national), justifiée par le souci, dans le projet global, d'identifier les pratiques d'enseignement efficaces auprès des élèves les plus fragiles.

Les caractéristiques sociales et scolaires des 2507 élèves accueillis dans les classes de l'échantillon sont elles aussi assez proches de la situation nationale, avec néanmoins une surreprésentation d'élèves de milieu social modeste (voir tableau 10 en annexe). Un peu moins de $4 \%$ des élèves redoublent leur CP à la rentrée 2013 (contre $3 \%$ pour la situation nationale) et un peu plus de $4 \%$ au total sont en retard scolaire (année de naissance inférieure à 2007). $72 \%$ des élèves parlent pas le français à la maison. Les $21 \%$ restants parlent plusieurs langues à la maison, dont le français. Enfin, on compte $50 \%$ de filles.

\section{Instruments de mesure et procédures}

Les élèves ont été évalués à l'entrée du cours préparatoire au mois de septembre dans différents domaines du lire-écrire ayant trait à la maîtrise du code graphophonologique (connaissance du nom des lettres, phonologie, lecture de mots et de pseudo-mots), à la compréhension (de phrases et de textes entendus), au vocabulaire, à l'écriture et au raisonnement non verbal. Une autre série d'épreuves a été administrée à la fin de l'année scolaire. Les épreuves sont détaillées dans le rapport de recherche (Goigoux, 2016, p. 56-81). Dans la mesure où l'objet de cet article ne porte pas sur les effets des aides sur les progressions des élèves, nous utiliserons seulement les résultats des élèves aux épreuves initiales, résultats qui nous permettent de situer chaque élève par rapport aux autres et par rapport à la moyenne de la distribution qui a été centrée-réduite (moyenne $=0$; écart-type $=1$ ).

Pour conduire certaines analyses, nous avons considéré comme "élèves les plus en difficulté» ceux dont le score à l'entrée au cours préparatoire est inférieur à un écart-type sous la moyenne (soit les $15 \%$ les plus faibles). Certains des résultats présentés sont alors dépendants de ce seuil. Ce choix méthodologique, s'il comporte une part d'arbitraire, permet néanmoins d'examiner au niveau du cours préparatoire ce qu'il en est de l'aide apportée à la tranche d'élèves (aux alentours de $15 \%$ ) que l'on retrouvera probablement en difficulté en lecture dans les niveaux ultérieurs (Daussin, Keskpaik \& Rocher, 2011; De La Haye, Gombert, Rivière et al., 2011). On sait en effet combien les difficultés en lecture dès le CP sont susceptibles de persister au-delà de l'école primaire (Caille, 2004), et que, de l'aveu de l'institution même, "les élèves qui sont en difficulté dès leur entrée au CP le sont toujours, dans leur quasi-totalité, par la suite» (HCE, 2007, p. 2).

Enfin, à trois moments de l'année ${ }^{4}$, les enseignants ont nommé les élèves qui ont bénéficié d'un dispositif d'aide (APC, RASED et orthophoniste) et quantifié le volume d'aide reçu (APC et RASED seulement). II

4 Fin de période 1 : mi-novembre 2013, soit 10 semaines d'école depuis la rentrée; fin de période 2 : mi-mars 2014, soit 13 semaines d'école depuis la période précédente; fin de période 3 : mi-mai 2014, soit 7 semaines d'école depuis la période précédente. 
conviendra néanmoins d'être prudent quant aux estimations relatives aux orthophonistes, que l'on peut penser sous-estimées, car il est possible qu'un enseignant n'ait pas connaissance du suivi d'un de ses élèves ${ }^{5}$.

\section{Quantifier l'aide offerte aux élèves de cours préparatoire}

\section{Effectifs d'élèves accueillis dans les dispositifs}

Le tableau 1 présente les effectifs d'élèves ayant bénéficié de dispositifs d'aide aux différentes périodes de recueil de données de l'année scolaire 2013-2014.

C'est le dispositif des APC qui accueille le plus d'élèves $(n=658)$, soit $26 \%$ des élèves de l'échantillon,

5 Les orthophonistes sont soumis au secret professionnel (article L 4344-2 du Code de la santé publique). Les enseignants n'ont donc connaissance du suivi orthophonique que si les parents le leur mentionnent. Le contact entre les deux professionnels nécessite également leur accord. entre début septembre et fin mai. Un peu moins de la moitié des élèves engagés dans le dispositif (soit $12 \%$ de l'échantillon total) a fréquenté les APC pendant une seule période alors qu'un peu plus d'un quart (ou $7 \%$ de l'échantillon total) en a bénéficié tout au long de I'année, soit 30 semaines. Viennent ensuite les maîtres $E$ du RASED et les orthophonistes, qui accueillent les élèves dans des proportions similaires : durant l'enquête, $13 \%$ des élèves de l'échantillon $(n=318)$ ont été suivis par un maître $E$, tandis que $12 \%(n=311)$ ont été suivis par un orthophoniste. Les maîtres G, enfin, accueillent $5 \%$ des élèves ( $n=112$ élèves).

\section{Intensité des aides}

Nos données permettent d'examiner l'intensité des aides reçues à travers deux indicateurs : le volume horaire délivré pendant l'année et la présence dans les dispositifs aux trois périodes de l'enquête. Nous disposons alors d'un volume global que l'on pourra rapprocher d'un volume attendu présenté en introduction et d'un indice de la concentration de l'aide ou, au contraire, de son échelonnement sur l'année. Les orthophonistes étant extérieurs à l'école, il ne nous a pas été possible 
Tableau 2. Volume moyen de l'aide dans les différents dispositifs durant les 30 semaines de l'étude

\begin{tabular}{c|c|c|c|c|c|c|c}
\hline \multicolumn{2}{c}{ APC } & \multicolumn{2}{c}{ Maître E } & Maître G & Orthophoniste \\
\hline $\begin{array}{c}\text { Durée } \\
\text { moyenne } \\
\text { (en heures) }\end{array}$ & Écart-type & $\begin{array}{c}\text { Durée } \\
\text { moyenne } \\
\text { (en heures) }\end{array}$ & Écart-type & $\begin{array}{c}\text { Durée } \\
\text { moyenne } \\
\text { (en heures) }\end{array}$ & Écart-type & $\begin{array}{c}\text { Durée } \\
\text { moyenne }\end{array}$ & Écart-type \\
\hline 11,2 & 9,8 & 20,1 & 23,5 & 10,1 & 9,5 & - & - \\
\hline
\end{tabular}

de recueillir le volume horaire d'aide dispensée aux élèves auprès de leurs enseignants. La communication entre les enseignants et les orthophonistes n'étant pas institutionnalisée, elle dépend en effet du bon vouloir et des possibilités des acteurs en présence.

En moyenne, les élèves participent aux APC à hauteur de 11,2 h (écart-type $=9,8$ ), ils bénéficient de l'aide du maître $E$ à hauteur de 20,1 h (écart-type $=23,5$ ) et de celle du maître $\mathrm{G}$ à hauteur de 10,1 h (écart-type $=9,5$ ) durant les 30 semaines de l'enquête. Autour de ces moyennes, il y a donc des variations importantes, comme en témoigne la figure 1 en annexe détaillant la répartition des heures d'APC. L'examen des effectifs cumulés de cette distribution nous permet par exemple de constater que, parmi les élèves aidés, la moitié a été aidée $8 \mathrm{~h}$ ou moins et les trois-quarts $15 \mathrm{~h}$ ou moins. À l'inverse, il apparaît que seulement un quart des élèves concernés par le dispositif $(6 \%$ de l'échantillon total) a été aidé plus de 15 h et $11 \%$ (soit $3 \%$ de l'échantillon total) ont reçu 25 h d'aide ou plus ${ }^{6}$.

Les données indiquent également que $25 \%$ des élèves aidés en APC ont fréquenté ce dispositif de manière échelonnée à l'année puisque leur présence a été signalée aux trois périodes du recueil de données. $31 \%$ des élèves ont été signalés à deux périodes et $44 \%$ à une période. Globalement, plus le nombre de

6 Les seuils ( 8,15 et 25 h) utilisés dans la suite des analyses auraient pu être différents, définis arbitrairement. Nous avons délimité des seuils qui peuvent être lus sur une échelle temporelle différente, hebdomadaire. Ainsi, 7-8 heures annuelles correspondent, sur la période de l'enquête, à une moyenne d'un quart $\mathrm{d}$ 'heure hebdomadaire. Quinze heures annuelles correspondent à une moyenne d'une demi-heure hebdomadaire; ce seuil correspond également au volume d'ALT manquant aux élèves les plus faibles évoqué en introduction. Trente heures hebdomadaires correspondent à une moyenne d'une heure hebdomadaire. Nous avons choisi de réduire ce dernier seuil à 25 heures afin d'éviter une définition trop rigide qui exclurait par exemple les élèves ayant bénéficié de 28 ou 29 heures annuelles. Néanmoins, si une moyenne hebdomadaire constitue une référence lisible pour le lecteur, cet indicateur sous-entend que l'aide s'échelonne sur toute l'année. Or, ce n'est pas le cas pour une grande partie des élèves. C'est pourquoi la question de l'intensité est traitée à la fois du point de vue périodes de fréquentation augmente, plus le volume horaire délivré est important : les élèves présents sur une période ont reçu en moyenne un peu plus de $5 \mathrm{~h}$ d'aide, ceux présents sur deux périodes ont reçu en moyenne près de $13 \mathrm{~h}$ et ceux présents sur les trois périodes près de $20 \mathrm{~h}$. Parmi les élèves aidés plus de 15 h, environ $40 \%$ d'entre eux (soit $3 \%$ de l'échantillon total) ont reçu cette aide de manière concentrée, c'està-dire sur une ou deux périodes. Ceci s'explique sans doute par l'organisation des APC qui sont limitées à une heure par semaine dans l'emploi du temps des élèves, contrairement au RASED ou à l'orthophoniste.

L'analyse fait cependant apparaître une situation similaire en ce qui concerne l'intensité de l'aide apportée par le RASED. Comme pour les APC, les élèves aidés de manière intensive sont peu nombreux : environ un quart des élèves admis, soit $4 \%$ de l'échantillon total, a été aidé $25 \mathrm{~h}$ ou plus par un maître $E$ et/ou un maître G. À l'inverse, environ la moitié des élèves accueillis en RASED (entre 7 et $8 \%$ de l'échantillon total) a bénéficié de moins de $15 \mathrm{~h}$ annuelles. Enfin, parmi les élèves aidés par un maître $E$ plus de $15 \mathrm{~h}$ annuelles $(n=141)$, la moitié a reçu cette aide de manière échelonnée pendant toute l'année scolaire.

Ces analyses ne peuvent pas être reconduites pour quantifier l'aide orthophonique du fait de l'absence de données concernant le volume horaire. On signalera simplement à ce stade que $30 \%$ des élèves aidés en APC ont fréquenté ce dispositif de manière échelonnée à l'année. $31 \%$ des élèves ont été signalés à deux périodes et $39 \%$ à une période.

\section{Cumul et subsidiarité des aides}

Enfin, il convient de compléter cette étude sur l'intensité de l'aide en examinant dans quelle mesure les élèves bénéficient de plusieurs aides simultanées. Le tableau 3 offre une vue globale des configurations d'aides relevées au cours de l'enquête.

En considérant simultanément les différents types d'aide, il apparaît qu'un tiers de l'échantillon (33\%) a été 
Tableau 3. Cumul des aides reçues au cours de l'année 2013-2014

\begin{tabular}{|c|c|c|}
\hline Aides reçues & Effectifs & $\%$ de l'échantillon total $(\mathrm{N}=2507)$ \\
\hline \multicolumn{3}{|l|}{ Une aide } \\
\hline APC seulement & 291 & 12 \\
\hline RASED seulement & 65 & 3 \\
\hline Orthophoniste seulement & 71 & 3 \\
\hline Total & 427 & 17 \\
\hline \multicolumn{3}{|l|}{ Deux aides } \\
\hline APC + RASED & 159 & 6 \\
\hline APC + Orthophoniste & 90 & 4 \\
\hline RASED + Orthophoniste & 32 & 1 \\
\hline Total & 281 & 11 \\
\hline \multicolumn{3}{|l|}{ Trois aides } \\
\hline APC + RASED + Orthophoniste & 118 & 5 \\
\hline Total & 826 & 33 \\
\hline
\end{tabular}

aidé au cours de l'année : $17 \%$ des élèves ont bénéficié d'un seul dispositif d'aide (le plus souvent l'APC qui rassemble à elle seule $12 \%$ des élèves), $11 \%$ de deux dispositifs et $5 \%$ de trois dispositifs. Le cumul de plusieurs dispositifs est ainsi la règle pour près de la moitié des élèves aidés. Dans ces cas, le plus souvent, l'aide de l'enseignant dans le cadre des APC est cumulée avec une aide spécialisée (15\% des élèves de l'échantillon).

Une lecture un peu rapide de ces premières données nous invite à penser que l'affectation dans les dispositifs suit un principe de subsidiarité : une partie des élèves n'est aidée qu'en APC, une autre en APC complétée par des enseignants spécialisés. Cette lecture est à nuancer au regard des $7 \%$ d'élèves qui reçoivent uniquement une aide spécialisée. Par ailleurs, le principe de subsidiarité suppose qu'une aide d'un niveau inférieur soit proposée en amont d'une aide de niveau supérieur. Si nous n'avons pas de données permettant de saisir directement cette information, nous pouvons examiner la circulation des élèves dans les dispositifs au cours des périodes. Ainsi, parmi les élèves ayant été aidés en APC en première période, on compte 162 élèves ayant été également suivis par un maître $\mathrm{E}$, dont 129 (soit 80 \%) au cours de cette même période. Ce résultat renforce le constat de cumul simultané de dispositifs plutôt qu'une fréquentation selon le principe de subsidiarité.

\section{Synthèse}

Ces premières analyses permettent plusieurs constats sur lesquels nous reviendrons ultérieurement. Le pre- mier concerne l'ampleur de l'utilisation de dispositifs d'aide extérieurs à la classe puisqu'environ un tiers des élèves de l'échantillon est concerné. Parmi les élèves aidés, la moitié cumule au moins deux dispositifs. Ce recours à l'externalisation de l'aide est cependant tempéré par la relative faiblesse du volume horaire dispensé. Nous reviendrons plus amplement sur ces résultats dans la dernière section de cet article.

\section{Estimer l'adéquation de l'aide aux caractéristiques des élèves}

Cette section a pour objectif d'examiner les caractéristiques des élèves accueillis au sein des dispositifs. II s'agit de questionner l'adéquation entre le public visé par ces dispositifs et le public effectivement accueilli. En l'absence de protocole officiel définissant les critères d'admission au sein des dispositifs, nous considérerons essentiellement les indicateurs liés au niveau de performances des élèves. Si l'objectif de ces dispositifs est d'apporter une aide aux élèves en difficulté, le public visé en priorité devrait être composé d'élèves ayant les plus faibles niveaux de performances en début d'année ou ayant des caractéristiques susceptibles d'être associées à un faible niveau de performances.

\section{Caractéristiques sociodémographiques des élèves accueillis dans les dispositifs}

En début de scolarité, on sait que certaines caractéristiques sociodémographiques sont statistiquement 


\begin{tabular}{|c|c|c|c|c|c|c|c|c|c|}
\hline & \multirow[t]{2}{*}{$\mathbf{N}$} & \multicolumn{2}{|c|}{ APC } & \multicolumn{2}{|c|}{ Maître E } & \multicolumn{2}{|c|}{ Orthophoniste } & \multicolumn{2}{|c|}{ Les 3 aides } \\
\hline & & $\mathbf{n}$ & $\%$ & $\mathbf{n}$ & $\%$ & $\mathbf{n}$ & $\%$ & $\mathbf{n}$ & $\%$ \\
\hline Issus d'un milieu favorisé & 484 & 61 & 13 & 13 & 3 & 27 & 6 & 6 & 1 \\
\hline Issus d'un milieu intermédiaire & 701 & 164 & 23 & 56 & 8 & 84 & 12 & 30 & 4 \\
\hline Issus d'un milieu modeste & 1030 & 324 & 32 & 174 & 17 & 146 & 14 & 57 & 6 \\
\hline Issus d'un milieu très défavorisé & 247 & 97 & 39 & 71 & 29 & 50 & 20 & 24 & 10 \\
\hline Khi2 & & \multicolumn{2}{|c|}{$85,576^{* *}$} & \multicolumn{2}{|c|}{$132,226^{* *}$} & \multicolumn{2}{|c|}{$38,316^{* *}$} & \multicolumn{2}{|c|}{$152,088^{* *}$} \\
\hline $\begin{array}{l}\text { Parlent uniquement le français } \\
\text { à la maison }\end{array}$ & 1797 & 439 & 24 & 170 & 10 & 207 & 12 & 77 & 4 \\
\hline $\begin{array}{l}\text { Parlent le français et une autre } \\
\text { langue à la maison }\end{array}$ & 542 & 147 & 27 & 90 & 17 & 74 & 14 & 28 & 5 \\
\hline $\begin{array}{l}\text { Parlent seulement une langue } \\
\text { étrangère à la maison }\end{array}$ & 168 & 72 & 43 & 58 & 35 & 30 & 18 & 13 & 8 \\
\hline Khi2 & & \multicolumn{2}{|c|}{$27,225^{* *}$} & \multicolumn{2}{|c|}{$96,737^{* *}$} & \multicolumn{2}{|c|}{$6,670^{*}$} & \multicolumn{2}{|c|}{$63,532 * *$} \\
\hline Nés après 2007 (en avance) & 19 & 2 & 11 & 2 & 11 & 1 & 5 & 1 & 5 \\
\hline Nés entre janvier et avril & 730 & 154 & 21 & 66 & 9 & 53 & 7 & 15 & 2 \\
\hline Nés entre mai et août & 859 & 222 & 26 & 101 & 12 & 98 & 11 & 36 & 4 \\
\hline $\begin{array}{l}\text { Nés entre septembre et } \\
\text { décembre }\end{array}$ & 790 & 225 & 29 & 110 & 14 & 94 & 12 & 44 & 6 \\
\hline Nés avant 2007 (retard scolaire) & 106 & 54 & 51 & 39 & 37 & 65 & 61 & 22 & 21 \\
\hline Khi2 & & \multicolumn{2}{|c|}{$47,949 * *$} & \multicolumn{2}{|c|}{$66,147^{* *}$} & \multicolumn{2}{|c|}{$252,794^{* *}$} & \multicolumn{2}{|c|}{$173,689 * *$} \\
\hline Filles & 1260 & 315 & 25 & 143 & 11 & 119 & 9 & 46 & 4 \\
\hline Garçons & 1247 & 343 & 28 & 175 & 14 & 192 & 15 & 72 & 6 \\
\hline Khi2 & & \multicolumn{2}{|c|}{$2,033 n s$} & \multicolumn{2}{|c|}{$4,078^{*}$} & \multicolumn{2}{|c|}{$20,436^{* *}$} & \multicolumn{2}{|c|}{$13,370^{*}$} \\
\hline
\end{tabular}

Lecture : parmi les 484 élèves de milieu favorisé de l'échantillon, 61 (12,6\%) ont fréquenté les APC.

Note : * écarts significatifs au seuil de 0,$05 ;{ }^{* *}$ : écarts significatifs au seuil de 0,01 .

associées à la probabilité de rencontrer des difficultés dans les apprentissages (Mingat, 1991). D'une manière générale, en lecture, plus l'univers culturel familial de l'élève s'éloigne de celui de l'école, plus cette probabilité augmente. C'est le cas également pour les élèves en retard scolaire, nés en fin d'année civile ou les garçons. Le tableau 4 rend compte de la fréquentation des dispositifs en fonction du milieu social d'origine des élèves, de la langue parlée à la maison, de leur âge et de leur sexe.

À la lecture de ce tableau, on constate que, de manière attendue, plus les élèves sont statistiquement susceptibles de rencontrer des difficultés, plus la probabilité d'être accueillis dans un dispositif d'aide ou de cumuler les dispositifs d'aide est forte. En APC, les élèves issus de milieu très défavorisé sont ainsi, en pro- les élèves de milieu favorisé : $39 \%$ des élèves très défavorisés ont été accueillis contre $13 \%$ des élèves de milieu favorisé. Les élèves ne parlant pas le français à la maison ou ceux en retard scolaire sont également significativement surreprésentés. II n'y a en revanche pas de différence significative de fréquentation des APC selon le sexe des élèves.

Les données relatives à l'aide dispensée par les maîtres $\mathrm{E}$ et les orthophonistes comme celles relatives au cumul des trois aides affichent des tendances identiques, sauf en ce qui concerne le sexe des élèves, les garçons y étant davantage présents que les filles. On soulignera que les élèves en retard scolaire sont particulièrement représentés au sein des dispositifs : si près de $20 \%$ d'entre eux n'ont reçu aucune aide, $31 \%$ ont bénéficié d'une aide, $30 \%$ de deux aides et $21 \%$ de trois aides. 
Un examen plus approfondi des variations de la fréquentation en fonction des caractéristiques des élèves nous permet de révéler des logiques que l'on pourrait qualifier de spécialisation?. Par exemple, les élèves très défavorisés sont quatre fois plus représentés que les élèves de milieu favorisé dans l'aide par les APC ou l'orthophoniste; ils le sont dix fois plus dans I'aide par un maître $E$. Le lien entre le milieu social et le type d'aide est donc plus faible pour les APC et l'orthophoniste que pour le maître $E$, ce qui signifie que les milieux favorisés et intermédiaires notamment sont

7 Pour plus de rigueur dans l'examen des probabilités d'admission au sein des différents dispositifs en fonction des caractéristiques des élèves, l'estimation de modèles probabilistes aurait été nécessaire (Desjardins, 2005; Bressoux, 2010). En effet, de simples analyses bivariées ne prennent pas en compte les liens entre les différentes caractéristiques. Par exemple, le milieu social des élèves est-il un facteur qui agit de manière autonome, au-delà de l'influence du niveau scolaire, de la langue parlée à la maison? De telles analyses multivariées permettraient donc un raisonnement «toutes choses égales par ailleurs». Elles sont cependant délicates à estimer ici, car de nombreux élèves fréquentent plusieurs dispositifs en même temps, ce qui empêche de déceler les logiques propres à tel ou tel dispositif. Une solution serait d'estimer ces modèles sur les élèves qui ne fréquentent qu'un seul dispositif, mais nous serions confrontés ici à un problème d'effectifs (42 élèves sont accueillis seulement par un maître E, 71 par un orthophoniste seulement) et donc de robustesse statistique. À titre exploratoire, ces modèles ont toutefois été estimés et produisent des résultats similaires à ceux obtenus avec les analyses bivariées. davantage présents en APC ou chez l'orthophoniste que chez le maître E. Les élèves ne parlant pas le français à la maison sont davantage surreprésentés chez les maîtres E. Les élèves en retard scolaire et les garçons le sont chez les orthophonistes.

Les données précédentes nous confirment que plus les élèves affichent des caractéristiques statistiquement liées à la probabilité de rencontrer des difficultés d'apprentissages, plus ils fréquentent les dispositifs étudiés ici. Toutefois, tous les élèves ayant ces caractéristiques n'éprouvent pas de difficultés. II convient donc maintenant de s'interroger sur le niveau scolaire des élèves accueillis et d'établir dans quelle mesure ce sont effectivement les élèves les plus faibles qui sont accueillis dans les différents dispositifs.

\section{Niveau scolaire à l'entrée au CP des élèves accueillis en APC}

Il s'agit d'examiner ici la modulation de la durée d'aide en fonction du niveau des élèves. Un premier examen des corrélations obtenues indique que plus les élèves ont des scores faibles aux épreuves de début d'année, plus la fréquentation du dispositif est intensive (voir tableaux 11 et 12 en annexe). Ce sont les résultats des élèves à l'épreuve de code qui semblent les plus déterminants, suivis par les résultats à l'épreuve de 
compréhension, puis ceux à l'épreuve d'écriture. Toutefois, les relations sont modestes, aux alentours de $-0,30$, ce qui indique une certaine dispersion que le tableau 5 permet de saisir.

Ce tableau appelle plusieurs constats. D'une part, il apparaît qu'un tiers des élèves faibles et les deux tiers des élèves très faibles sont accueillis en APC. C'est le cas d'un peu plus de $10 \%$ des bons élèves et de $3 \%$ des très bons. Rappelons qu'un quart des élèves de l'échantillon a été accueilli en APC, il apparaît donc clairement que la proportion des élèves faibles et très faibles à être accueillis en APC est supérieure à la moyenne. À l'inverse, les deux tiers des élèves faibles, et plus du tiers des élèves très faibles, n'y sont pas accueillis. D'autre part, en examinant les volumes horaires distribués, parmi les élèves les plus faibles, un quart a été accueilli plus de $15 \mathrm{~h}$ au cours de l'année. Enfin, on peut remarquer que si un certain nombre d'élèves très faibles ne sont pas accueillis en APC $(n=149)$, une quantité similaire d'élèves ayant un niveau supérieur à la moyenne de l'échantillon en début d'année y est accueillie $(n=118)$.

Enfin, alors que notre étude se déroule au moment charnière où les APC viennent de prendre le relais de l'aide personnalisée, elle montre que ce dispositif est encore principalement investi par les enseignants comme un dispositif d'aide. En effet, une seule classe accueille successivement tous ses élèves en APC et $9 \%$ des classes n'en accueillent aucun. Sans doute ces classes investissent les heures dédiées aux APC à d'autres desseins que l'aide aux élèves en difficulté, tel qu'autorisé par les textes réglementaires dans le cadre d'une «activité prévue par le projet d'école » (MENESR, 2013b). Par ailleurs, d'une part, seuls $3 \%$ des «bons» élèves (plus d'un écart-type au-dessus de la moyenne) y sont accueillis, pour quelques heures. D'autre part, si l'analyse de la fréquentation des APC en fonction des caractéristiques du contexte scolaire ne peut être développée ici, on constate que le nombre d'élèves concernés par les APC dans chaque classe est d'autant plus élevé que le nombre d'élèves faibles et très faibles y est important. Les APC sont ainsi majoritairement demeurées un dispositif d'aide aux élèves.

\section{Niveau scolaire à l'entrée au CP des élèves aidés par un maître $E$}

Les tableaux 13 et 14 en annexe nous indiquent une relation significative entre les performances initiales c'est le tableau 6 qui nous permet d'apprécier plus précisément l'adéquation de l'intensité de l'aide du maître $E$ avec le niveau des élèves.

II en ressort que $85 \%$ des élèves faibles et plus de $60 \%$ des élèves très faibles n'ont pas été aidés par un maître E. Parmi les $39 \%$ d'élèves très faibles aidés, la moitié a bénéficié de moins de $15 \mathrm{~h}$ d'aide au cours des 30 semaines d'enquête. D'un autre côté, relativement peu d'élèves qui n'affichaient pas de performances particulièrement faibles en début d'année y sont accueillis : $4 \%$ des bons élèves et un seul très bon élève ont été aidés par un maître $E(n=35)$. Contrairement aux APC, les situations d'inadéquation entre le niveau scolaire des élèves et l'aide du maître $E$ ne sont pas à chercher du côté des bons élèves qui n'y auraient pas leur place. En revanche, parmi les élèves faibles, les plus faibles ne sont pas systématiquement ceux qui font l'objet de ce type d'aide.

Enfin, lorsque l'analyse de la fréquentation est portée au niveau des caractéristiques du contexte, on constate que le nombre d'élèves aidés par un maître $E$ dans chaque classe est d'autant plus élevé que le nombre d'élèves faibles y est important, mais également que la proportion d'élèves de milieu défavorisé ou ne parlant pas le français à la maison est forte. Ainsi, les maîtres $E$ aident en moyenne 1,7 élève par classe hors éducation prioritaire contre 4,2 en moyenne par classe en éducation prioritaire $(F=22,99$, au seuil de $0,000)$.

\section{Niveau scolaire à l'entrée au CP des élèves aidés par un orthophoniste}

Les données relatives à la consultation d'un orthophoniste sont des données déclaratives recueillies auprès des enseignants, mode de recueil qui implique un certain nombre de limites déjà évoquées dans cet article. Le temps passé par les élèves chez l'orthophoniste n'étant pas connu, c'est l'examen du nombre de périodes pendant lesquelles les élèves ont été suivis qui se fait en fonction de leur niveau à l'entrée au CP. II ne s'agit donc pas d'un indicateur de durée tel qu'il a été proposé dans les sections précédentes. L'intensité est ici uniquement appréhendée par la longueur du suivi sur l'année scolaire (voir tableau 15 en annexe et tableau 7).

Les constats sont ici similaires aux précédents qui concernaient les maîtres $\mathrm{E}$. Si environ $15 \%$ des élèves faibles sont aidés par un orthophoniste, c'est le cas d'un tiers des élèves très faibles. Les bons et très bons 


\begin{tabular}{|c|c|c|c|c|c|c|c|c|}
\hline \multirow{3}{*}{$\begin{array}{l}\text { Durée } \\
\text { de la fréquentation }\end{array}$} & \multicolumn{8}{|c|}{ Élèves dont le score global est... } \\
\hline & \multicolumn{2}{|c|}{$\begin{array}{l}\text { Inférieur à } \\
\text { la moyenne -1 } \\
\text { écart-type } \\
(\mathrm{N}=383)\end{array}$} & \multicolumn{2}{|c|}{$\begin{array}{l}\text { Compris entre } \\
\text { la moyenne et }-1 \\
\text { écart-type } \\
(\mathrm{N}=885)\end{array}$} & \multicolumn{2}{|c|}{$\begin{array}{l}\text { Compris entre } \\
\text { la moyenne }+1 \\
\text { écart-type } \\
(\mathrm{N}=840)\end{array}$} & \multicolumn{2}{|c|}{$\begin{array}{l}\text { Supérieur à } \\
\text { la moyenne + } 1 \\
\text { écart-type } \\
(N=399)\end{array}$} \\
\hline & $\mathbf{n}$ & $\%$ & $\mathbf{n}$ & $\%$ & $\mathbf{n}$ & $\%$ & $\mathbf{n}$ & $\%$ \\
\hline $\begin{array}{l}\text { Élèves non accueillis } \\
(n=2189)\end{array}$ & 234 & 61 & 751 & 85 & 806 & 96 & 398 & 100 \\
\hline $\begin{array}{l}\text { Élèves ayant bénéficié } \\
\text { de moins de } 8 h \quad(n=79)\end{array}$ & 33 & 9 & 34 & 4 & 11 & 1 & 1 & 0 \\
\hline $\begin{array}{l}\text { Élèves ayant bénéficié } \\
\text { de } 8 \text { à } 15 \mathrm{~h}(\mathrm{n}=98)\end{array}$ & 35 & 9 & 46 & 5 & 9 & 1 & 0 & 0 \\
\hline $\begin{array}{l}\text { Élèves ayant bénéficié } \\
\text { de plus de } 15 h(n=141)\end{array}$ & 81 & 21 & 54 & 6 & 14 & 2 & 0 & 0 \\
\hline $\begin{array}{l}\text { Toutes durées confondues } \\
(n=318)\end{array}$ & 149 & 39 & 134 & 15 & 34 & 4 & 1 & 0 \\
\hline
\end{tabular}

élèves sont aidés dans une bien moindre proportion (respectivement $5 \%$ et $3 \%$ ). Parmi les élèves les plus faibles, un tiers a été aidé tout au long de l'année et, d'une manière générale, plus les élèves étaient faibles en début d'année, plus le suivi a été durable sur l'année. Néanmoins, on peut à nouveau souligner que de nombreux élèves très faibles en début d'année, un peu moins des deux tiers $(n=249)$, n'ont pas bénéficié de ce type d'aide alors que, paradoxalement, d'autres élèves meilleurs qu'eux en ont bénéficié sur les trois périodes examinées $(n=176$, dont 50 élèves avec des scores initiaux supérieurs à la moyenne). L'adéquation entre le niveau des élèves et l'existence d'un suivi orthophonique n'est ainsi que partielle.

\section{Cumul et subsidiarité des aides pour les élèves les plus faibles}

Afin de déterminer dans quelle mesure les élèves les plus en difficulté bénéficient des différents dispositifs d'aide, nous examinons ici la situation des 383 élèves (15\% de l'échantillon total) qui présentaient les performances les plus faibles à l'entrée au cours préparatoire, à savoir inférieures à un écart-type sous la moyenne.

La multiplicité des aides apparaît liée au niveau scolaire affiché par les élèves en début d'année (voir tableau 16 en annexe) : en moyenne, plus les élèves présentent des performances faibles en début d'année, plus ils se voient proposer de dispositifs d'aide. Mais le tableau 8 présente un bilan mitigé.
Il apparaît ainsi que $25 \%$ des élèves les plus en difficulté n'ont bénéficié d'aucun dispositif d'aide. En ajoutant à ces derniers les $5 \%$ d'élèves qui ont été aidés seulement par un orthophoniste, ce sont alors $30 \%$ des élèves les plus faibles qui n'ont bénéficié d'aucune aide de la part de l'institution scolaire, qu'il s'agisse des APC ou de l'aide d'un maître du RASED. Plus d'un quart n'a bénéficié que d'un seul dispositif (le plus souvent I'APC qui rassemble à elle seule $17 \%$ des élèves très faibles). Un tiers a bénéficié de deux dispositifs et $16 \%$ des trois. Près de la moitié des élèves (49\%) a donc cumulé plusieurs dispositifs d'aide, celle de l'enseignant assortie d'un spécialiste dans la grande majorité des cas.

À nouveau, une lecture un peu rapide de ces premières données nous invite à penser que l'affectation dans les dispositifs suit un principe de subsidiarité : une partie des élèves n'est aidée qu'en APC, une autre en APC complétée par un ou des spécialistes. Mais il convient de souligner les $14 \%$ d'élèves qui sont uniquement aidés par un spécialiste. Et, parmi les élèves très faibles ayant été aidés en APC en première période, on compte 89 élèves ayant été également suivis par un maître E pendant l'année, dont 74 (soit $83 \%$ ) au cours de cette même période. De la même manière que sur l'ensemble des élèves accueillis, c'est davantage une logique de cumul simultané de dispositifs qui semble caractériser la fréquentation des élèves les plus faibles que l'application du principe de subsidiarité.

À partir du moment où de nombreux élèves cumulent les dispositifs, il reste à examiner les volumes 


\begin{tabular}{|c|c|c|c|c|c|c|c|c|}
\hline \multirow{3}{*}{ Durée de la fréquentation } & \multicolumn{8}{|c|}{ Élèves dont le score global est... } \\
\hline & \multicolumn{2}{|c|}{$\begin{array}{l}\text { Inférieur à } \\
\text { la moyenne -1 } \\
\text { écart-type } \\
(\mathrm{N}=383)\end{array}$} & \multicolumn{2}{|c|}{$\begin{array}{l}\text { Compris entre } \\
\text { la moyenne et }-1 \\
\text { écart-type } \\
(\mathrm{N}=885)\end{array}$} & \multicolumn{2}{|c|}{$\begin{array}{l}\text { Compris entre } \\
\text { la moyenne }+1 \\
\text { écart-type } \\
(\mathrm{N}=840)\end{array}$} & \multicolumn{2}{|c|}{$\begin{array}{c}\text { Supérieur à } \\
\text { la moyenne + } 1 \\
\text { écart-type } \\
(\mathrm{N}=399)\end{array}$} \\
\hline & $\mathrm{n}$ & $\%$ & $\mathrm{n}$ & $\%$ & $\mathbf{n}$ & $\%$ & $\mathrm{n}$ & $\%$ \\
\hline Élèves non accueillis ( $\mathrm{n}=2196$ ) & 249 & 65 & 758 & 85,6 & 802 & 95,5 & 387 & 97,0 \\
\hline $\begin{array}{l}\text { Élèves accueillis sur } \\
\text { une période }(n=122)\end{array}$ & 44 & 12 & 53 & 6 & 16 & 2 & 9 & 2 \\
\hline $\begin{array}{l}\text { Élèves accueillis sur } \\
2 \text { périodes }(n=95)\end{array}$ & 44 & 12 & 40 & 6 & 9 & 1 & 2 & 1 \\
\hline $\begin{array}{l}\text { Élèves accueillis sur } \\
3 \text { périodes }(n=94)\end{array}$ & 46 & 12 & 34 & 4 & 13 & 2 & 1 & 0 \\
\hline $\begin{array}{l}\text { Toutes périodes confondues } \\
(\mathrm{n}=311)\end{array}$ & 135 & 35 & 127 & 14 & 38 & 5 & 12 & 3 \\
\hline
\end{tabular}

Tableau 8. Nombre d'élèves en difficulté bénéficiant de dispositifs d'aide

\begin{tabular}{l|c|c}
\hline \multirow{2}{*}{} & \multicolumn{2}{c}{ Élèves les plus en difficulté ayant reçu... } \\
\cline { 2 - 3 } & \multicolumn{2}{|c}{$\mathbf{n}$} \\
\hline Aucune aide & 96 & 25 \\
\hline Une aide & 65 & 17 \\
\hline APC uniquement & 16 & 4 \\
RASED uniquement & 19 & 5 \\
Orthophoniste uniquement & 100 & 26 \\
Total & \multicolumn{2}{|c}{} \\
\hline Deux aides simultanées & 72 & 19 \\
\hline APC + RASED & 37 & 10 \\
APC + Orthophoniste & 18 & 53 \\
RASED + orthophoniste & 127 & 16 \\
Total & \multicolumn{2}{|c}{} \\
\hline Trois aides simultanées & 383 & 100 \\
\hline APC + RASED + Orthophoniste & \multicolumn{2}{|c}{} \\
\hline Total &
\end{tabular}




\begin{tabular}{|c|c|c|c|c|c|c|}
\hline & \multicolumn{3}{|c|}{ Élèves tous niveaux confondus } & \multicolumn{3}{|c|}{ Élèves très faibles } \\
\hline & $\mathrm{n}$ & $\begin{array}{c}\% \text { parmi } \\
\text { l'échantillon } \\
\text { total } \\
\mathrm{N}=2507\end{array}$ & $\begin{array}{l}\% \text { parmi } \\
\text { les élèves } \\
\text { accueillis } \\
\mathrm{N}=755\end{array}$ & $\mathrm{n}$ & $\begin{array}{c}\% \text { parmi } \\
\text { l'échantillon } \\
\text { total } \\
\mathrm{N}=383\end{array}$ & $\begin{array}{c}\% \text { parmi } \\
\text { les élèves } \\
\text { accueillis } \\
N=268\end{array}$ \\
\hline $\begin{array}{l}\text { Élèves ayant bénéficié } \\
\text { de moins de } 8 \mathrm{~h} \text { d'aide }\end{array}$ & 257 & 10 & 34 & 56 & 15 & 21 \\
\hline $\begin{array}{l}\text { Élèves ayant bénéficié } \\
\text { de } 8 \text { à } 15 \text { h d'aide }\end{array}$ & 168 & 7 & 22 & 41 & 11 & 15 \\
\hline $\begin{array}{l}\text { Élèves ayant bénéficié } \\
\text { de plus de } 15 \text { h d'aide } \\
\text { (dont } 25 \text { h et plus) }\end{array}$ & $\begin{array}{r}330 \\
(217)\end{array}$ & $\begin{array}{l}13 \\
(9)\end{array}$ & $\begin{array}{r}44 \\
(29)\end{array}$ & $\begin{array}{r}171 \\
(124)\end{array}$ & $\begin{array}{r}45 \\
(32)\end{array}$ & $\begin{array}{r}64 \\
(46)\end{array}$ \\
\hline Total & 755 & 30 & 100 & 268 & 70 & 100 \\
\hline
\end{tabular}

horaires cumulés - à l'exclusion de l'aide orthophonique, dont les durées nous sont inconnues - afin de savoir si les élèves les plus faibles bénéficient en moyenne d'une aide plus importante en termes de volume horaire. En sommant le volume respectif déclaré pour chaque aide, nous obtenons une image globale de l'aide proposée au cours préparatoire en 2013-2014 pour les 755 élèves (dont 268 élèves très faibles) qui ont été reçus en APC et/ou en RASED (voir tableau 9).

Le cumul des heures d'aides reçues par les élèves, APC et RASED confondus, permet d'estimer que $17 \%$ des élèves de l'échantillon ont bénéficié de moins de 15 h d'aide annuelle. À l'inverse, 13 \% d'entre eux ont été aidés plus de $15 \mathrm{~h}$ annuelles (et $9 \% 25 \mathrm{~h}$ ou plus). Lorsque l'on porte l'analyse sur les élèves les plus en difficulté, environ un quart (26\%) a reçu moins de $15 \mathrm{~h}$ annuelles et une petite moitié ( $45 \%$ ) a reçu plus de $15 \mathrm{~h}$ annuelles. Un tiers (32\%) a été aidé plus de $25 \mathrm{~h}$ durant les 30 semaines de l'étude. Une autre lecture de ce tableau nous indique que parmi les 217 élèves aidés plus de $25 \mathrm{~h}, 124$ sont des élèves très en difficulté à l'entrée en CP mais 93 ne comptent pas parmi les plus en difficulté. Dans le même temps, 259 comptant parmi les plus en difficulté en ont reçu moins, voire pas du tout. À nouveau, l'adéquation entre le niveau des élèves et le volume d'aide dont ces derniers bénéficient est partielle.

\section{Synthèse}

Le principal résultat mis en évidence dans cette section concerne la relative inadéquation entre les caractéris- tiques sociodémographiques et scolaires du public visé par les dispositifs et les caractéristiques du public effectivement accueilli. Certes, ce sont essentiellement les élèves faibles qui fréquentent les dispositifs, mais le volume d'aide dispensée peut être considéré comme modeste et de nombreux élèves parmi les plus faibles ne sont pas aidés quand d'autres, moins faibles en début d'année scolaire, le sont. Un deuxième résultat est relatif à la présence d'une logique de cumul de dispositifs, déjà mise en évidence précédemment, mais qui s'avère concerner davantage les élèves les plus faibles que les autres élèves aidés. Enfin, nos données semblent révéler une logique de spécialisation : au-delà du niveau scolaire des élèves, déterminant de la fréquentation des trois dispositifs examinés, les élèves de milieux très défavorisés et ne parlant pas le français à la maison sont davantage accueillis par les maîtres $E$, les élèves redoublants et les garçons davantage par l'orthophoniste.

\section{Discussion}

Le temps consacré aux apprentissages est un des facteurs fondamentaux du niveau d'acquisition des élèves. Ce constat est désormais bien établi (Crahay, 2012), et des travaux plus récents se sont attachés non seulement à en produire des mesures fines, basées sur l'engagement réel des élèves, mais également à apprécier la distance entre ce qui est offert aux élèves et ce dont ils auraient besoin pour afficher, plus tard, de bonnes performances (Suchaut, Bougnères \& Bouguen, 2014). Pour combler le décalage qui apparaît en particulier chez les élèves les plus fragiles, lesquels ont 
besoin de davantage de temps que les autres, une stratégie consiste à augmenter les temps consacrés aux apprentissages par la mise en place de dispositifs spécifiques. Notre objectif a été de saisir dans quelle mesure les dispositifs d'aide offerts aux élèves sont susceptibles de combler ce décalage.

Certes, nos analyses présentent quelques limites. Nous avons souligné que notre échantillon, bien que proche de la situation nationale, n'en est pas strictement représentatif, notamment du fait d'une surreprésentation des élèves scolarisés en éducation prioritaire. Ce biais limite la possibilité de généralisation des résultats descriptifs notamment. On peut ainsi supposer que nos estimations sont surestimées si les aides sont plus fréquentes là où les élèves en difficulté sont plus concentrés. Nos données confirment d'ailleurs que les élèves de l'éducation prioritaire sont plus nombreux à recevoir de l'aide de leur enseignant dans le cadre des APC ou des enseignants spécialisés des RASED (ce n'est pas le cas des orthophonistes). S'il est possible que la situation décrite soit, en termes d'aide, légèrement plus favorable que la situation nationale, les constats établis dans ce travail sur le nombre important d'élèves faibles non aidés ou peu aidés n'en sont que plus marquants.

\section{Intensité de l'aide aux élèves en difficulté au cours préparatoire}

D'une manière générale, si l'on prend en compte l'aide proposée par le système scolaire (les APC et l'aide pédagogique du RASED), un tiers des élèves de cours préparatoire a été aidé au cours des 30 semaines de l'enquête. Environ un quart des élèves de l'échantillon a bénéficié des APC, et moins de $15 \%$ d'une aide spécialisée. Est-ce beaucoup? Est-ce peu ? Pour éviter une réponse normative, il est possible de comparer les caractéristiques de l'aide française avec celle apportée dans d'autres pays qui présentent moins d'élèves en difficulté - c'est le cas de la Finlande d'après l'enquête PISA (OCDE, 2014) - ou avec celle apportée par des dispositifs d'aide validés par la recherche. À titre de comparaison donc, en Finlande, $25 \%$ des élèves de l'école primaire bénéficient d'une aide spécialisée, dans des écoles dont la grande majorité dispose d'un enseignant spécialisé (Takala, Pirttimaa \& Törmänen, 2009) qui cible son action sur les élèves de cours préparatoire (Takala \& Haustätter, 2012).

Le niveau de difficulté des élèves est positivement le volume d'aide reçu est important et plus les différents types de dispositifs d'aide se cumulent. Si I'on additionne APC et RASED, un peu moins de $10 \%$ des élèves de l'échantillon ont bénéficié d'un volume d'aide supérieur à 25 heures annuelles. Parmi les $15 \%$ des élèves les plus faibles en début d'année, un tiers a bénéficié de plus de 25 heures annuelles. Enfin, un volume horaire élevé va souvent de pair avec une fréquentation tout au long de I'année scolaire. Or, la recherche semble souligner la meilleure efficacité d'aides intensives (plusieurs séances hebdomadaires) sur une période resserrée, inférieure ou égale à 20 semaines (Elbaum, Vaughn, Hughes et al., 2000). La plupart des dispositifs d'aide américains proposent une intervention quotidienne d'une durée comprise entre 20 et 50 minutes (Wanzek \& Vaughn, 2008), comme le programme Reading Recovery (Viriot-Goeldel, 2011). Ainsi les aides proposées aux élèves français sont-elles moins spécialisées et surtout moins intensives que d'autres types d'aides proposées à l'étranger dont la recherche a validé l'efficacité.

Par ailleurs, parmi les $15 \%$ des élèves les plus faibles en début d'année, un tiers n'a pas été accueilli dans les dispositifs d'aide du cadre scolaire (APC et RASED). Un peu plus de la moitié de ces élèves faibles n'a pas bénéficié d'une aide spécialisée (RASED). Certes, ces élèves ont pu être aidés par leur enseignant en classe dans le cadre d'une différenciation ordinaire. Mais l'aide de l'enseignant ne peut être assimilée à l'aide d'un enseignant spécialisé et c'est cette différence qui apparaît entre les niveaux 1 et 2 de la typologie évoquée en introduction ou dans les instructions officielles qui établissent la subsidiarité entre l'aide de l'enseignant et celle du RASED. Supprimer le niveau 2 de l'aide spécialisée au profit de l'aide en classe n'est d'ailleurs pas le choix fait par exemple au Canada et aux États-Unis, où les élèves peuvent bénéficier d'aide spécialisée en plus de l'aide en classe (Viriot-Goeldel, 2007, 2011), ou encore en Finlande, où des chercheurs attribuent les bons résultats de leur pays dans les enquêtes internationales à ce système d'aide spécialisée (Takala, Pirttimaa \& Törmänen, 2009).

Les estimations que nous produisons ici sont à mettre en parallèle avec la baisse des effectifs d'enseignants spécialisés des RASED amorcée à partir de 2005. Selon les sources (IGEN, 2013; Cour des comptes, 2015), les chiffres peuvent différer légèrement, mais on estime que les emplois implantés dans les RASED ont été diminués d'un tiers environ depuis le milieu des années 2000. Par ailleurs, les aides apportées par le maître de la classe 
ont récemment diminué de moitié dans le cadre du passage à la rentrée 2013 d'une aide personnalisée de deux heures hebdomadaires aux APC de $36 \mathrm{~h}$ annuelles, soit $1 \mathrm{~h}$ hebdomadaire. Réduits en volume, ces temps se sont aussi élargis à d'autres activités que le soutien aux élèves en difficulté, affaiblissant peut-être la lisibilité de ce dispositif. Sans doute cette double situation concourt-elle à expliquer le nombre important d'élèves faibles non aidés dans le cadre scolaire (30\%) et le faible volume horaire de l'aide en général.

Enfin, en cohérence avec le principe de subsidiarité, selon lequel l'aide devrait d'abord être apportée au niveau de l'échelon de responsabilité le plus proche, c'est-à-dire par le maître de la classe, ce sont les APC qui accueillent le plus d'élèves (aux alentours de $25 \%$ des élèves de l'échantillon), les RASED et les orthophonistes en accueillant deux fois moins environ (respectivement $15 \%$ et $12 \%)$. Cependant, ce principe de subsidiarité ne se trouve pas complètement respecté car tous les élèves ayant bénéficié d'une aide spécialisée, que l'on peut qualifier de niveau 2, n'ont pas auparavant bénéficié des $A P C$. La relative inadéquation de l'aide aux difficultés des élèves est également mise en évidence à travers le fait que certains élèves faibles en début d'année n'ont pas été aidés, et que d'autres élèves, moins en difficulté, l'ont été. La question des critères d'attribution de l'aide se pose donc de manière aiguë, interrogeant tant le fonctionnement des dispositifs que les représentations des acteurs. En effet, à la baisse des emplois affectés en RASED s'ajoute la «sédentarisation » des maîtres $E$, qui n'interviennent plus toujours sur l'ensemble de leur circonscription. L'enquête de la Cour des comptes établit que les maîtres E n'interviennent que dans $55 \%$ des écoles (Cour des comptes, 2015), excluant de fait les élèves des autres écoles. À l'inverse, les APC fonctionnent dans toutes les écoles, mais le volume fixé ne tient pas compte des caractéristiques scolaires des élèves. Un enseignant dispose d'une heure hebdomadaire quel que soit le nombre d'élèves en difficulté dans sa classe. Dans les classes où les élèves en difficulté sont peu nombreux, un enseignant pourra élargir les critères d'attribution quand, dans une autre classe où les élèves en difficulté sont plus nombreux, un enseignant pourra les réduire ou procéder à des arbitrages tels que de recourir à d'autres aides pour certains élèves, instaurant une certaine division du travail sur laquelle nous revenons plus loin. En l'absence de procédure définie et de critères institutionnels pour désigner les bénéficiaires des différents types d'aide, I'admission des élèves dans les différents dispositifs d'aide semble relever de plusieurs logiques qui coexistent, sans que nous ne puissions déterminer avec certitude les intentions des acteurs en présence.

\section{L'aide aux élèves en difficulté : témoin des transformations de l'organisation scolaire}

Au-delà de la quantification de l'aide, il semble possible d'inscrire nos résultats dans un ensemble de travaux décrivant les transformations contemporaines de l'organisation scolaire, en particulier à travers la multiplication des dispositifs dans et hors de la sphère scolaire.

L'ampleur du nombre d'élèves aidés par des dispositifs dans notre échantillon témoigne de la fin d'un certain modèle scolaire, homogène et uniforme (Barrère, 2013b). Résultat d'un processus engagé depuis le début des années 1980, où la mise en œuvre de logiques différenciatrices devait permettre d'en finir avec l'indifférence aux différences, le recours aux dispositifs permet à la fois une individualisation et une inclusion, mots d'ordre de la prescription institutionnelle de l'aide (Cauterman \& Daunay, 2016). Ils permettent "de "faire autrement" avec "certains", voire avec "chacun" [...] se multipliant ainsi afin de ne pas déroger à une logique d'action qui reste universaliste » (Barrère, 2013b, p. 106).

Le recours aux dispositifs est bien à l'heure actuelle un «outil essentiel de la gestion d'un certain nombre de difficultés scolaires» (Moignard \& Rubi, 2013, p. 58). En la matière, on retrouve dans nos données deux des trois pôles d'intervention professionnelle de l'échec scolaire décrit par S. Morel (2012) : le pôle pédagogique (APC et maître E du RASED) et le pôle médical (orthophonique). L'ampleur du recours aux APC est assez inattendue. Un élève sur quatre y participe alors qu'une enquête récente sur la réception des APC par les enseignants (Harris Interactive, 2015) souligne I'insatisfaction de ces derniers à l'égard de ce dispositif, jugé fatigant pour les élèves en difficulté et considéré comme moins approprié que d'autres dispositifs. On se trouve ici peut-être devant le paradoxe que génère ce qu'Alter (2000, cité par Barrère, 2013b) qualifie d'invention dogmatique : les $\mathrm{APC}$ constituent une réponse à un besoin d'action en même temps que s'affichent des résistances, n'étant pas vraiment utilisées différemment de l'aide personnalisée qu'elles sont censées remplacer depuis 2013. À l'inverse, la tendance à un recours important aux orthophonistes dans le cas des difficultés en lecture largement pressentie dans 
plusieurs études qualitatives (Morel, 2014; Viriot-Goeldel, 2007) se trouve ici confirmée. Ce recours concerne plus d'un élève sur dix et avoisine celui des RASED. II prend place dans un double mouvement de croissance des effectifs de la profession (+ $38 \%$ entre 2001 et 2010; Observatoire national de la démographie des professions de santé [ONDPS], 2012) et d'affaiblissement de l'offre de remédiation pédagogique évoqué plus haut.

Loin de se concurrencer, le pôle pédagogique et le pôle médical ont plutôt tendance à se cumuler. Tout se passe comme si les dispositifs permettaient non seulement une réponse à l'urgence de ne pas laisser s'installer des difficultés scolaires précoces mais également une possibilité de mobiliser plusieurs types de remédiation dans un contexte où l'efficacité de chacune est loin d'être démontrée (Barrère, 2013b).

\section{Externalisation de l'aide et division du travail}

Plus largement, avec un élève sur trois qui fréquente au moins un des dispositifs étudiés, l'externalisation de la prise en charge de la difficulté scolaire par l'école contemporaine n'est plus seulement une tendance (Moignard \& Rubi, 2013). L'idée paraît bien établie «que la classe en soi n'est pas le lieu le mieux adapté pour l'aide individuelle» (Cauterman \& Daunay, 2010, p. 12-13), alors même les politiques d'inclusion scolaire en font plus que jamais le lieu de scolarisation de tous les élèves. La division du travail mise en évidence entre le scolaire et l'éducatif (Moignard \& Rubi, 2013; Tardif \& Levasseur, 2015) s'observe en fait à l'intérieur même du champ strictement scolaire. On assiste ici à une triple externalisation, un peu différente de celle mise en évidence par Cauterman et Daunay (2010). Un premier niveau se situe hors la classe (non au sens physique mais comme cadre instituant) avec l'enseignant titulaire dans le cadre des APC. Lorsque les difficultés sont plus intenses ou plus spécifiques, les deux autres niveaux sont mobilisés : hors la classe avec les enseignants spécialisés des RASED ou hors l'école avec les orthophonistes.

Cette externalisation, surtout lorsqu'elle mobilise des spécialistes hors l'école, pose la question de la cohérence des jugements et des diagnostics posés sur les élèves et de l'existence d'une continuité scolaire qui, si elle est un enjeu institutionnellement affiché, peut ne pas toujours être une priorité (Chartier, 2013; Moignard \& Rubi, 2013). L'impossibilité dans notre recherche d'appréhender avec certitude le nombre de suivis orthophoniques et le volume horaire de ce suivi témoigne, à l'instar de Morel (2014), d'un déficit de communication entre l'école et les orthophonistes. Le travail réalisé en classe est, de fait, déconnecté de celui réalisé chez l'orthophoniste, là où la recherche recommande une «coordination [...] au niveau de l'enseignement, afin que les élèves bénéficient de méthodes qui soient synergétiques, et non fragmentées» (Snow, Burns \& Griffin, 1998, p. 333). Dans ces conditions, on peut craindre que le suivi orthophonique n'influe guère sur la situation de l'élève en difficulté en classe.

\section{La médicalisation de l'échec scolaire}

La banalisation de l'externalisation de l'aide aux élèves en difficulté s'accompagne d'une médicalisation de l'échec scolaire. À l'œuvre dans de nombreux pays, dont les pays anglo-saxons qui ont créé le terme de «reading disability» et la France depuis la fin des années 1970 (Pinell \& Zafiropoulos, 1978), ce mouvement découle sans doute d'un faisceau de causes historiques, scientifiques et pragmatiques dont il serait trop long de faire état ici (voir notamment Garcia, 2013; Garcia \& Oller, 2015 ; Morel, 2014; Viriot-Goeldel, 2013). À ce propos, on remarque que parmi les élèves qui ont consulté un orthophoniste durant l'année, la moitié n'a pas vu de maître E du RASED. Autrement dit, il n'a pas été tenté de leur apporter une aide spécialisée pédagogique dans le cadre scolaire avant d'externaliser leur suivi. Pour d'autres, le suivi orthophonique vient en complément d'une aide à l'école dont l'intensité ne semble pas à la hauteur des aides validées par la recherche à l'étranger.

Médicaliser la remédiation des difficultés de lecture soulève un certain nombre d'interrogations. Transférer une partie de sa mission première - faire en sorte que chaque élève sache lire et écrire - à un tiers hors de l'école apparaît comme un «dédouanement » de l'institution scolaire, a fortiori lorsqu'elle n'a pas tenté d'apporter elle-même une aide spécialisée aux élèves en question. Ce transfert lui évite ainsi une remise en cause de ses propres pratiques, qu'il s'agisse des pratiques pédagogiques de l'enseignant ou du fonctionnement des dispositifs d'aide. Il est à ce propos assez marquant de voir à quel point ce sont les élèves redoublants qui sont les plus concernés par le recours aux orthophonistes. Cela signe-t-il l'échec de l'institution qui mobilise d'autres registres explicatifs des difficultés pour ces élèves qui ne correspondent pas à son 
modèle pédagogique (Moignard \& Rubi, 2013)? Qui s'exonère de la responsabilité de leur échec? Cela est-il le fruit d'une mobilisation des parents pour qui «l'étiquetage des difficultés est souvent un moment pivot» (Morel, 2012, p. 158) légitimant leur intervention dans la recherche de traitements alternatifs et diagnostics différents de celui de l'école, déculpabilisant envers eux-mêmes et leur enfant puisque médical ? Nos données ne nous permettent pas de trancher, mais donnent à voir une réaction nette à l'égard d'un mode de traitement institutionnel de la difficulté scolaire dont l'efficacité est largement contestée (Paul, 1996; Troncin, 2005). Par ailleurs, l'école délègue le soutien des élèves en difficulté à un corps professionnel ne partageant pas la même conception de la lecture, ni, par conséquent, la même approche rééducative (Morel, 2014), posant à nouveau la question de la continuité éducative. Enfin, en matière de médicalisation, on conçoit, outre le caractère prescripteur du médecin ou de l'enseignant, le poids de l'implication parentale dans une telle démarche (voir notamment Garcia, 2013; Morel, 2012). Nécessitant un ensemble de ressources que les parents les plus démunis ne possèdent pas, le recours aux orthophonistes légèrement plus important dans les milieux plus favorisés invite à souligner le risque d'accroissement des inégalités entre élèves.

\section{Conclusion}

Nos analyses ont permis de produire ici un certain nombre de chiffres inédits sur l'aide aux élèves en difficulté, dans un domaine où «le simple dénombrement de l'existant est problématique» (Cour des comptes, 2015). Elles ont fait apparaître que de nombreux élèves faibles (30\%) ne recevaient aucune aide dans le cadre scolaire, et que l'adéquation entre le niveau des élèves et la participation à des dispositifs d'aide était plus qu'imparfaite. Insuffisants en matière d'intensité, les dispositifs d'aide accueillent néanmoins de nombreux élèves de cours préparatoire (environ un tiers).

Nos résultats témoignent, avec d'autres, d'un mouvement général d'externalisation de l'aide hors de la classe et hors de l'école, à travers le développement de dispositifs depuis le début des années 1990 (Morel, 2014) et plus encore au cours des années 2000, où se multiplient les prescriptions institutionnelles en matière de soutien scolaire, de lutte contre le décrochage ou d'inclusion des élèves en situation de handicap (Zay, 2012; Barrère, 2013a). L'externalisation et la médicalisation du traitement de la difficulté posent la question du morcellement de l'action éducative (Moignard \& Rubi, 2013), de la possibilité de définir un réel partenariat entre des dispositifs dont la multiplicité risque d'être contradictoire et contre-productive. La question de l'efficacité de ces aides est en effet cruciale. En France, plusieurs études sur le sujet n'ont pas permis de conclure à l'efficacité des aides spécialisées à l'école (Mingat \& Richard, 1991 ; Piquée, 2007).

Nos analyses invitent alors à réexaminer cette question des effets des aides. Les données ne permettent pas de rechercher les effets de chacun des dispositifs, car le nombre important d'élèves concernés par plusieurs dispositifs rend illusoire toute tentative d'évaluation : il serait impossible d'attribuer ces effets, positifs ou négatifs, à tel ou tel dispositif. Par ailleurs, en l'état actuel de la littérature sur ce sujet, des informations sur la nature des aides dispensées (contenu, continuité pédagogique et transfert des acquisitions dans le quotidien des apprentissages en classe dans le cas des aides externalisées, etc.) seraient nécessaires afin de démêler si les effets sont attribuables à des causes structurelles ou à des contenus plus ou moins appropriés. En revanche, la question des effets du cumul de dispositifs pourrait être explorée : alors que certains élèves faibles ne bénéficient d'aucun dispositif, d'autres les additionnent. Ce cumul constitue-t-il une réponse pertinente au doute sur l'efficacité des dispositifs pris un à un? À l'inverse, risque-t-il de faire de l'élève accueilli un «sujet fragmenté, à qui est interdit le statut d'élève, au profit de l'entrée dans une carrière de bénéficiaires et d'usagers de dispositifs multiples, qui l'éloignent un peu plus encore des exigences de l'école» (Moignard \& Rubi, 2013, p. 59)?

Céline Piquée

Université Rennes 2, CREAD celine.piquee@univ-rennes2.fr

Caroline Viriot-Goeldel Université Paris Est, EA 4384 Université Paris 8-Vincennes-Saint-Denis, CIRCEFT, UPEC caroline.viriot-goeldel@u-pec.fr 


\section{Bibliographie}

ALTER N. (2000). L'innovation ordinaire. Paris : PUF.

BARRÈRE A. (2013a). «Les établissements scolaires à I'heure des "dispositifs"». Carrefours de l'éducation, n`36, p. 9-13.

BARRÈRE A. (2013b). «La montée des dispositifs : un nouvel âge de l'organisation scolaire». Carrefours de l'éducation, $\mathrm{n}^{\circ} 36, \mathrm{p} .95-116$

BONJOUR E. \& GOMBERT J.-É. (2004). «Profils de lecteurs à l'entrée en sixième». L'Orientation scolaire et professionnelle, vol. 33, ${ }^{\circ}{ }^{1}$, p. 69-101.

BRESSOUX P. (2010). Modélisation statistique appliquée aux sciences sociales. Bruxelles : De Boeck, $2^{\mathrm{e}}$ éd.

CAILLE J.-P. (2004). «Le redoublement à l'école élémentaire et dans l'enseignement secondaire : évolution des redoublements et parcours scolaires des redoublants au cours des années 1990-2000». Éducation et formations, n 69, p.79-88.

CARROLL J. (1963). «A model of school learning». Teachers college record, vol. 64, $\mathrm{n}^{\circ}$ 8, p.723-733.

CAUTERMAN M.-M. \& DAUNAY B. (2010). «La jungle des dispositifs ». Recherches, n 52 , p. 9-23.

CAUTERMAN M.-M. \& DAUNAY B. (2016). «La prescription institutionnelle de l'aide : mots d'ordre et désordres». Recherches, $\mathrm{n}^{\circ}$ 64, p.7-23.

CHARTIER M. (2013). «L'accueil des enfants du voyage : d'un dispositif académique à son appropriation par les acteurs éducatifs des écoles élémentaires». Carrefours de l'éducation, $\mathrm{n}^{\circ} 36, \mathrm{p} .31-45$.

CHATEL L. (2010). «Plan pour prévenir l'illettrisme et susciter le goût de la lecture». Discours prononcé le 29 mars 2010. En ligne : <http://www.education.gouv.fr/cid50954/prevention-de-l-illettrisme.html> (consulté le 18 septembre 2015).

CHOPIN M.-P. (2010). "Les usages du "temps" dans les recherches sur l'enseignement ». Revue française de pédagogie, $\mathrm{n}^{\circ} 170$, p. 87-110.

COLMANT M., DAUSSIN J.-M. \& BESSONNEAU P. (2011). «Compréhension de l'écrit en fin d'école. Évolutions de 2003 à 2009». Note d'information de la DEPP, n¹1-16.

COUR DES COMPTES (2015). Le suivi individualisé des élèves : une ambition à concilier avec l'organisation du système éducatif. Paris : La Documentation française.

CRAHAY M. (dir.) (2012). L'école peut-elle être juste et efficace? Bruxelles : De Boeck.

DAUSSIN J.-M., KESKPAIK S. \& ROCHERT. (2011) L'évolution du nombre d'élèves en difficulté face à l'écrit depuis une dizaine d'années. France, portrait social, 2011. En ligne : <http:// www.insee.fr/fr/statistiques/1373895?sommaire=1373905> (consulté le 20 avril 2015).

DE LA HAYE F., GOMBERT J.-É., RIVIÈRE J.-P. \& VOURC'H R. (2011). «Les évaluations en lecture dans le cadre de la Journée Défense et Citoyenneté. Année 2010 ». Note d'information de la DEPP, $\mathrm{n}^{\circ} 11-28$.
DESJARDIN J. (2005). «L'analyse de régression logistique». Tutorial in Quantitative Methods for Psychology, vol. 1, $\mathrm{n}^{\circ}$ 1, p.35-41.

ELBAUM B., VAUGHN S., HUGHES M., \& MOODY S. (2000). "How effective are one-to-one tutoring programs in reading for elementary students at risk for reading failure? A meta-analysis of the intervention research ». Journal of Educational Psychology, n 92, p.605-619.

FERRY L., DARCOS X. \& HAIGNERÉ C. (2003). Lettre à tous ceux qui aiment l'école : pour expliquer les réformes en cours. Paris : Odile Jacob.

FUCHS D. \& FUCHS L. S. (2006). «Introduction to Response to Intervention: What, Why, and How Valid Is It? ». Reading Research Quarterly, vol. 41, n¹, p. 93-99.

GAMBERT P. \& BONNEAU J. (2005). Portrait des enseignants du premier degré : interrogation de 1000 enseignants du premier degré en mai-juin 2004. Paris : Ministère de l'Éducation nationale, de l'Enseignement supérieur et de la Recherche, DEPP. En ligne : <http://media.education.gouv. fr/file/49/6/2496.pdf> (consulté le 18 septembre 2015).

GARCIA S. (2013). À l'école des dyslexiques. Naturaliser ou combattre l'échec scolaire. Paris : La Découverte.

GARCIA S. \& OLLER A.-C. (2015). Réapprendre à lire. Paris : Éd. du Seuil.

GIBERT F., LEVASSEUR J. \& PASTOR J.-M. (2004). «La maîtrise du langage et de la langue française en fin d'école primaire». Note d'évaluation de la Depp, n 04-10.

GOIGOUX (dir.) (2016). «Lire-Écrire. Étude de l'influence des pratiques d'enseignement sur la qualité des premiers apprentissages ». Rapport de recherche pour l'Institut français de l'Éducation (École normale supérieure de Lyon) et le Ministère de l'Éducation nationale, de l'Enseignement supérieur et de la Recherche.

HAAGER D., KLINGER J. \& VAUGHN S. (dir.) (2007). Evidence-Based Reading Practices for Response to Intervention. Baltimore : Paul H. Brookes Publishing Co.

HARRIS INTERACTIVE (2015). Les préoccupations des enseignants de primaire. Vague 2. Enquête pour le Syndicat national unitaire des instituteurs, professeurs des écoles et PEGC (SNUipp). En ligne : <http://www.snuipp.fr/IMG/pdf/Note_ Harris_-_Les_preoccupations_des_enseignants_en_2015_ SNUipp_.pdf>(consulté le 18 août 2016)

HAUT CONSEIL DE L'ÉDUCATION (2007). L'école primaire. Bilan des résultats de l'école - 2007. En ligne : <http://www.hce. education.fr/gallery_files/site/21/40.pdf> (consulté le 20 avril 2015).

HAUT CONSEIL DE L'ÉDUCATION (2012). Rapport 2012. En ligne: $<$ http://www.ladocumentationfrancaise.fr/var/storage/rapports-publics/124000658.pdf> (consulté le 20 avril 2015).

INSPECTION GÉNÉRALE DE L'ÉDUCATION NATIONALE (2013). Les réseaux d'aides spécialisées aux élèves en difficulté 
- RASED - Rôles et perspectives d'évolution. Rapport n²013042.

INTERNATIONAL ASSOCIATION FOR THE EVALUATION OF EDUCATIONAL ACHIEVEMENT (2012). PIRLS 2011 International Results in Reading. Boston; Amsterdam : IEA.

MINISTÈRE DE L'ÉDUCATION, DU LOISIR ET DU SPORT (2011). Référentiel d'intervention en lecture pour les élèves de 10 à 15 ans. Québec : gouvernement du Québec.

MINISTÈRE DE L'ÉDUCATION NATIONALE, DE L'ENSEIGNEMENT SUPÉRIEUR ET DE LA RECHERCHE (2013a). «Prévenir I'illettrisme». Circulaire n²013-179 du 12 novembre 2013. Bulletin officiel de l'Éducation nationale, de l'Enseignement supérieur et de la Recherche, n 43, 21 novembre 2013.

MINISTÈRE DE L'ÉDUCATION NATIONALE, DE L'ENSEIGNEMENT SUPÉRIEUR ET DE LA RECHERCHE (2013b). «Organisation du temps scolaire dans le premier degré et des activités pédagogiques complémentaires» Circulaire $n^{\circ}$ 2013-017 du 6 février 2013. Bulletin officiel de l'Éducation nationale, de l'Enseignement supérieur et de la Recherche, $\mathrm{n}^{\circ}$ 6, 7 février 2013.

MINISTÈRE DE L'ÉDUCATION NATIONALE, DE L'ENSEIGNEMENT SUPÉRIEUR ET DE LA RECHERCHE (2014a). «Adaptation scolaire et scolarisation des élèves handicapés. Fonctionnement des réseaux d'aides spécialisées aux élèves en difficulté (RASED) et missions des personnels qui y exercent ». Circulaire n²014-107 du 18 août 2014. Bulletin officiel de l'Éducation nationale, de l'Enseignement supérieur et de la Recherche, n³1, 28 août 2014.

MINISTÈRE DE L'ÉDUCATION NATIONALE, DE L'ENSEIGNEMENT SUPÉRIEUR ET DE LA RECHERCHE (2014b). Repères et références statistiques. Paris : Ministère de l'Éducation nationale, de l'Enseignement supérieur et de la Recherche, DEPP.

MINGAT A. (1991). «Expliquer la variété des acquisitions au cours préparatoire : les rôles de l'enfant, la famille et l'école». Revue française de pédagogie, n95, p.47-63.

MINGAT A. \& RICHARD M. (1991). «Évaluation des activités de Rééducation GAPP à l'école primaire». Cahier de I'IREDU, $\mathrm{n}^{\circ} 49$.

MOIGNARD B. \& RUBI S. (2013). «Des dispositifs pour les élèves perturbateurs : les collèges à l'heure de la sous-traitance?». Carrefours de l'éducation, n³6, p.47-60.

MOREL S. (2012). «La cause de mon enfant». Politix, n99, p.153-176.

MOREL S. (2014). La médicalisation de l'échec scolaire. Paris : La Dispute.

OCDE (2014). PISA 2012 Results: What Students Know and Can Do - Student Performance in Mathematics, Reading and Science (Volume I, Revised edition, February 2014). Paris : PISA, OECD Publishing. En ligne: <http://www.oecd.org/ pisa/keyfindings/pisa-2012-results-volume-l.pdf> (consulté le 18 septembre 2015).

OBSERVATOIRE NATIONAL DE LA DÉMOGRAPHIE DES PROFESSIONS DE SANTÉ (2012). Étude sur les champs d'interventions des orthophonistes. Les spécificités et les coopérations avec d'autres professions. Paris : ONDPS. En ligne :
<http://social-sante.gouv.fr/IMG/pdf/Etude_sur_le_ metier_d_orthophoniste.pdf> (consulté le 28 juillet 2016).

PAUL J.-J (1996). Le redoublement. Pour ou contre? Paris : ESF.

PINELL P. \& ZAFIROPOULOS M. (1978). «La médicalisation de l'échec scolaire. De la pédopsychiatrie à la psychanalyse infantile». Actes de la recherche en sciences sociales, vol. 24, $\mathrm{n}^{\circ} 1, \mathrm{p} .23-49$.

PIQUÉE C. (2007). «Effets des pratiques à l'égard des élèves en difficulté au cours préparatoire». Actes du congrès international de l'Actualité de la recherche en éducation et en formation (AREF), Strasbourg. En ligne : <http://www. congresintaref.org/actes_pdf/AREF2007_Celine_ PIQUEE_221.pdf> (consulté le 18 septembre 2015).

ROCHER T. (2008). «Lire, écrire, compter : les performances des élèves de CM2 à vingt ans d'intervalle 1987-2007». Note d'information de la Depp, $\mathrm{n}^{\circ}$ 08-38.

ROCHER T. \& LE DONNÉ N. (2012). «Les difficultés de lecture en début de sixième Évolution à dix ans d'intervalle (19972007) ». Éducation et formations, $n^{\circ} 82$, p. 31-37.

SNOW C. E., BURNS M. S. \& GRIFFIN P. (dir.) (1998). Preventing reading difficulties in young children. Washington : National Academy Press.

SUCHAUT B. (1996). «La gestion du temps à l'école maternelle et primaire : diversité des pratiques et effets sur les acquisitions des élèves". L'Année de la recherche en sciences de l'éducation, p. 123-153.

SUCHAUT B., BOUGNÈRES A. \& BOUGUEN A. (2014). Sept minutes pour apprendre à lire. En ligne : <https://halshs. archives-ouvertes.fr/halshs-01062065/document> (consulté le 20 avril 2015).

TAKALA M. \& HAUSSTÄTTER S. (2012). «Effects of history and culture on attitudes toward special education: a comparison of Finland and Norway». International Scholarly Research Network. En ligne : <https://www.hindawi.com/ journals/isrn/2012/161039/> (consulté le 18 septembre 2015).

TAKALA M., PIRTTIMAA R. \& TÖRMÄNEN M. (2009). «Inclusive special education: the role of special education teachers in Finland». British Journal of Special Education, vol. 36, $\mathrm{n}^{\circ} 3$, p. 162-172.

TARDIF M. \& LEVASSEUR L. (2015). La division du travail éducatif : une perspective nord-américaine. Paris : PUF.

TRONCIN T. (2005). Le redoublement: radiographie d'une décision à la recherche de sa légitimité. Thèse de doctorat, sciences de l'éducation, université de Bourgogne.

VIRIOT-GOELDEL C. (2007). «Apprentis-lecteurs en difficulté : quels types d'aide? Essai d'analyse théorique et praxéologique de l'aide apportée aux élèves en difficulté dans les classes de l'enseignement primaire en France, au Québec et dans le Bade-Wurtemberg ". Carrefours de l'éducation, $\mathrm{n}^{\circ} 23, \mathrm{p} .25-38$.

VIRIOT-GOELDEL C. (2011). "Les programmes "clé en main" aux États-Unis : des modèles d'intervention pédagogique en réponse à la crise de l'école ». Actes du colloque AECSE Crise et/en éducation, Nanterre La Défense. 
VIRIOT-GOELDEL C. (2013). «Prévenir l'illettrisme dès l'école primaire : analyse du cas français à la lumière de la comparaison internationale». Cahiers de la recherche sur l'éducation et les savoirs, $n^{\circ} 12$, p. 59-70.

WANZEK J. \& VAUGHN S. (2008). «Response to varying amounts of time in reading intervention for students demonstrating insufficient response to intervention». Journal of Learning Disabilitie, vol. 41, n², p. 126-142.

ZAY D. (2012). L'Éducation inclusive. Une réponse à l'échec scolaire? Paris : L'Harmattan. 
Note : a : source MENESR, 2014b, p. 99, situation au cycle général du collège, les données pour l'école élémentaire ne sont pas disponibles ; $b$ : hors professeurs ; $c$ : professeurs, professeurs des écoles et instituteurs; $d$ : hors professeurs des écoles et instituteurs.

* : le recueil d'informations sur les professions des parents s'avère très délicat : certains enseignants se refusent à les connaître, d'autres à les communiquer. II a souvent été possible d'obtenir uniquement un codage direct à partir de la nomenclature en 8 postes des professions et catégories socioprofessionnelles (PCS) de l'INSEE. Lorsque le recueil n'était pas possible auprès des enseignants, un courrier a été adressé aux parents, les invitant à nous divulguer cette information, soit de manière précise, soit au sein de la nomenclature. Ayant demandé de renseigner la profession des deux parents, nous comptabilisons au final $2 \%$ de données manquantes. Un recodage a ensuite été effectué à partir de la profession la plus élevée des deux parents. La catégorie favorisée regroupe les élèves dont la profession la plus élevée, pour au moins un des deux parents, est cadre. La catégorie intermédiaire regroupe les élèves dont la profession la plus élevée, pour au moins un des deux parents, est agriculteur exploitant, artisan, commerçant, chef d'entreprises de 10 salariés ou plus ou de profession intermédiaire. La catégorie modeste regroupe les élèves dont la profession ou la situation la plus élevée, pour au moins un des deux parents, est employé, ouvrier ou retraité. La catégorie très défavorisée regroupe les élèves dont les parents sont sans emploi. Pour les parents sans emploi ou retraités, dans la mesure où l'ancienne profession a rarement été renseignée, nous avons examiné la distribution des scores de leurs enfants et la localisation de leur école. La moitié des élèves ayant un parent retraité et l'autre sans emploi est scolarisée dans une école relevant de l'éducation prioritaire. Leurs scores sont assez faibles, pour ceux relavant de l'éducation prioritaire comme pour les autres, les situant en moyenne en dessous des enfants d'ouvriers et au-dessus des enfants dont les deux parents sont sans emploi. Parmi ces derniers, près des deux tiers sont scolarisés dans une école relevant de l'éducation prioritaire. Ceux scolarisés hors du secteur éducation prioritaire ont des scores également en moyenne plutôt faibles : les deux tiers ont un score inférieur à la moyenne de l'échantillon et seuls $10 \%$ ont un score supérieur à un écart-type au-dessus de la moyenne $(n=10)$. Ces résultats nous ont conduits à classer les élèves dont les deux parents sont sans emploi dans la catégorie très défavorisée. Ceux ayant un parent retraité et l'autre sans emploi sont classés dans la catégorie modeste (c'est également cette catégorie qui compte le plus d'élèves et dans laquelle les faibles effectifs d'élèves dans cette situation perturberont le moins les estimations en cas d'erreur de classement). 


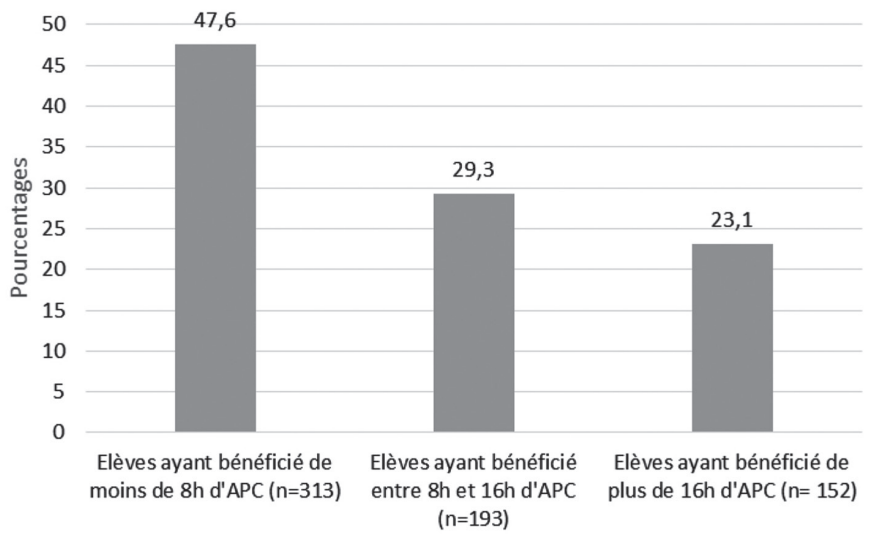

Tableau 11. Corrélation entre les scores des élèves en début d'année et le temps passé en APC

\begin{tabular}{|c|c|c|c|c|}
\hline & Écriture & Code & Compréhension & Score global \\
\hline $\begin{array}{l}\text { Temps d'accueil en APC } \\
\text { (pour l'ensemble des élèves de l'échantillon; } \\
\mathrm{N}=2507 \text { ) }\end{array}$ & $-0,289 * *$ & $-0,342^{* *}$ & $-0,311^{* *}$ & $-0,393 * *$ \\
\hline $\begin{array}{l}\text { Temps d'accueil en APC } \\
\text { (pour l'ensemble des élèves accueillis en APC; } \\
N=658 \text { ) }\end{array}$ & $-0,184^{* *}$ & $-0,239 * *$ & $-0,228^{* *}$ & $-0,292^{* *}$ \\
\hline
\end{tabular}

** : corrélation significative au seuil de 0,01.

Tableau 12. Scores en début d'année en fonction de la durée de fréquentation des APC

\begin{tabular}{l|c}
\hline Durée de fréquentation des APC & Score moyen (écart-type) \\
\hline Élèves non accueillis $(\mathrm{n}=1849)$ & $0,26(0,92)$ \\
\hline Élèves ayant bénéficié de moins de $8 \mathrm{~h}$ d'APC $(\mathrm{n}=343)$ & $-0,55(0,83)$ \\
\hline Élèves ayant bénéficié de 8 à $16 \mathrm{~h}$ d'APC $(\mathrm{n}=163)$ & $-0,69(0,79)$ \\
\hline Élèves ayant bénéficié de plus de $16 \mathrm{~h}$ d'APC $(\mathrm{n}=152)$ & $-1,14(0,82)$ \\
\hline Toutes durées confondues $(\mathrm{n}=658)$ & $-0,72(0,85)$ \\
\hline
\end{tabular}


Tableau 13. Corrélation entre les scores des élèves en début d'année et le temps passé avec un maître $\mathrm{E}$

\begin{tabular}{|c|c|c|c|c|}
\hline & Écriture & Code & Compréhension & Score global \\
\hline $\begin{array}{l}\text { Temps d'aide avec un maître } E \\
\text { (pour l'ensemble des élèves de l'échantillon; } \\
N=2507 \text { ) }\end{array}$ & $-0,158^{* *}$ & $-0,232^{* *}$ & $-0,209^{* *}$ & $-0,290^{* *}$ \\
\hline $\begin{array}{l}\text { Temps d'aide avec un maître } E \\
\text { (pour l'ensemble des élèves aidés par un } \\
\text { maître } E ; N=318 \text { ) }\end{array}$ & $-0,110^{*}$ & $-0,171^{* *}$ & $-0,186^{* *}$ & $-0,242^{* *}$ \\
\hline
\end{tabular}

Note: ${ }^{*}$ : corrélation significative au seuil de 0,$05 ;{ }^{* *}$ : corrélation significative au seuil de 0,01.

Tableau 14. Scores en début d'année en fonction de la durée de l'aide dispensée par un maître $\mathrm{E}$

\begin{tabular}{l|c}
\hline Durée de fréquentation du RASED (maître E) & Score moyen (écart-type) \\
\hline Élèves non accueillis $(\mathrm{n}=1849)$ & $0,14(0,94)$ \\
\hline Élèves ayant bénéficié de moins de $8 \mathrm{~h}(\mathrm{n}=343)$ & $-0,87(0,87)$ \\
\hline Élèves ayant bénéficié de 8 à $16 \mathrm{~h}(\mathrm{n}=163)$ & $-0,88(0,80)$ \\
\hline Élèves ayant bénéficié de plus de $16 \mathrm{~h}(\mathrm{n}=152)$ & $-1,11(0,81)$ \\
\hline Toutes durées confondues $(\mathrm{n}=658)$ & $-0,98(0,83)$ \\
\hline
\end{tabular}

Tableau 15. Scores en début d'année en fonction de la durée du suivi par un orthophoniste

\begin{tabular}{l|c}
\hline Score global & Score moyen (écart-type) \\
\hline Élèves non accueillis $(\mathrm{n}=2196)$ & $0,11(0,96)$ \\
\hline Élèves accueillis sur une période $(\mathrm{n}=122)$ & $-0,61(0,83)$ \\
\hline Élèves accueillis sur deux périodes $(\mathrm{n}=95)$ & $-0,92(0,94)$ \\
\hline Élèves accueillis sur trois périodes $(\mathrm{n}=94)$ & $-0,94(0,87)$ \\
\hline Toutes durées confondues $(\mathrm{n}=311)$ & $-0,81(0,89)$ \\
\hline
\end{tabular}

Tableau 16. Comparaison des scores moyens en début d'année en fonction de nombre d'aides reçues

\begin{tabular}{l|c|c|c|c}
\hline $\begin{array}{l}\text { Nombre d'aides } \\
\text { reçues }\end{array}$ & $\begin{array}{c}\text { Score moyen en } \\
\text { écriture }\end{array}$ & $\begin{array}{c}\text { Score moyen en } \\
\text { code }\end{array}$ & $\begin{array}{c}\text { Score moyen en } \\
\text { compréhension }\end{array}$ & Score moyen global \\
\hline Aucune & 0,25 & 0,31 & 0,26 & 0,34 \\
\hline Une & $-0,43$ & $-0,49$ & $-0,29$ & $-0,49$ \\
\hline Deux & $-0,61$ & $-0,73$ & $-0,78$ & $-0,89$ \\
\hline Trois & $-0,54$ & $-0,90$ & $-0,86$ & $-1,01$ \\
\hline
\end{tabular}

Note : tous les écarts sont significatifs au seuil de 0,000. 